\title{
OPERATORS WITH INVERSES SIMILAR TO THEIR ADJOINTS
}

\author{
U. N. SINGH AND KANTA MANGLA
}

\begin{abstract}
AbSTRACr. If $T$ is an invertible operator on a Hilbert space such that $S^{-1} T^{-1} S=T^{*}$ and $0 \notin \mathrm{Cl}(W(S))$ for some invertible operator $S$, where $\mathrm{Cl}(W(S))$ denotes the closure of the numerical range of $S$ and $T^{*}$ is the adjoint of $T$, then it is shown that $T$ is similar to a unitary operator. In fact, this has been proved as a corollary to a more general result, which also includes the corresponding result of J. P. Williams for selfadjoint operators.
\end{abstract}

Introduction. A selfadjoint operator $T$ on a Hilbert space $H$ is one for which $T^{*}=T$, where $T^{*}$ denotes the adjoint of $T$. However, if $T$ is only similar to $T^{*}$, then a result due to J. P. Williams [4] states that under certain conditions $T$ turns out to be similar to a selfadjoint operator. This provides a motivation to prove the corresponding result for unitary operators. In fact, an operator $T$ is called unitary if $T T^{*}=I=T^{*} T$, i.e. if $T^{-1}$ exists and $T^{-1}=T^{*}$. One of the objects of this paper is to show that if $T$ is an invertible operator for which $T^{-1}$ is similar to $T^{*}$, then under certain very natural restrictions $T$ is similar to a unitary operator, and if, in addition, $T$ is normaloid, then $T$ is unitary.

We shall denote by $W(T)$ the numerical range of $T: W(T)=\{(T x, x)$ : $\|x\|=1\}$ and by $\mathrm{Cl}(W(T))$ the closure of $W(T)$. A unitary operator $U$ is called cramped if its spectrum $\sigma(U)$ is contained in some open semicircle $\left\{e^{i \theta}: \theta_{0}<\theta<\theta_{0}+\pi\right\}$ of the unit circle [2].

Preliminary Remark. If $P$ is a + ve invertible operator and if $T P^{2}=$ $P^{2} T^{*}$ then $P^{-1} T P=P T^{*} P^{-1}=$ selfadjoint. Similarly the condition $T^{-1} P^{2}=$ $P^{2} T^{*}$ implies that $P^{-1} T^{-1} P=P T^{*} P^{-1}=$ unitary. Hence $T$ is similar to a selfadjoint operator (to a unitary operator) if and only if $T$ and $T^{*}$ are conjugate ( $T^{-1}$ and $T^{*}$ are conjugate) by means of a positive invertible operator. The converse assertions follow by polar decomposing the operator effecting the conjugacy of $T$ and $T^{*}$ (of $T^{*}$ and $T^{-1}$ ).

These ideas motivate the following theorem which, in fact, is inherent in the proof of Theorem 2 of [4].

Received by the editors October 18, 1971 and, in revised form, July 31, 1972.

AMS (MOS) subject classifications (1969). Primary 4730, 4755; Secondary 4615.

Key words and phrases. Hilbert space, selfadjoint operators, unitary operators, normaloid operators, numerical range.

(c) American Mathematical Society 1973 
THEOREM 1. If $J$ is a linear operator on $B(H)$, the Hilbert space of all bounded linear operators on $H$, such that $J\left(X^{*}\right)=J(X)^{*}$ for all $X \in B(H)$ then $J(S)=0$ for some $S$ such that $0 \notin \mathrm{Cl}(W(S))$ if and only if $J(P)=0$ for some positive invertible $P$.

Proof. Let $J(S)=0$ for some $S$ with $0 \notin \mathrm{Cl}(W(S))$. Since $0 \notin \mathrm{Cl}(W(S))$ and $\mathrm{Cl}(W(S))$ is convex [3, Problem 166] by replacing $S$ by $S e^{i \theta}$, if necessary, we can separate 0 from $W(S)$ by a halfplane. We choose $\theta$ such that the halfplane is $\operatorname{Re} z \geqq \varepsilon$ for some $\varepsilon>0$. If $A=\left(S+S^{*}\right) / 2$ then it is easy to see that $W(A)=\operatorname{Re} W(S)$. This implies that $W(A)$ lies on the real axis. Also for each $\lambda \in W(A), \lambda \geqq \varepsilon$. This $A$ is positive and invertible. Now since $J$ is linear

$$
J(A)=\frac{1}{2}\left[J(S)+J(S)^{*}\right]=0 .
$$

The converse of this is obviously true.

We have the following important corollaries:

Corollary 1 (Williams [2, Theorem 2]). If $S^{-1} T S=T^{*}$ where $0 \notin \mathrm{Cl}(W(S))$, then $T$ is similar to a selfadjoint operator.

The converse of this is also true, i.e. if $T$ is similar to a selfadjoint operator, then $T$ and $T^{*}$ are conjugate by an $S$ with $0 \notin \mathrm{Cl}(W(S))$.

COROLlaRY 2. If an invertible operator $T$ is such that $S^{-1} T^{-1} S=T^{*}$ where $0 \notin \mathrm{Cl}(W(S))$, then $T$ is similar to a unitary operator.

The converse of this is also true, i.e. if an invertible operator $T$ is similar to a unitary operator then $T^{*}$ and $T^{-1}$ are conjugate by an operator $S$ with $0 \notin \mathrm{Cl}(W(S))$.

For the proof of these corollaries it suffices to take

$$
J(X)=i\left(T X-X T^{*}\right) \text { and } \quad J(X)=T X T^{*}-X, \quad \text { respectively. }
$$

COROLlaRY 3. If $T$ is an invertible normaloid operator such that $T^{*}=S^{-1} T^{-1} S, 0 \notin \mathrm{Cl}(W(S))$, then $T$ is unitary.

Proof. A normaloid operator with spectrum on the unit circle is unitary.

THEOREM 2. If $T$ is an operator such that $T^{*}=U^{*} T^{-1} U$, where $U$ is a cramped unitary operator, then $T$ is unitary.

Proof. From $T^{*}=U^{*} T^{-1} U$ we have

$$
U T^{*}=T^{-1} U \text {. }
$$

Now by taking the inverses, we get $U T^{*-1}=T U$. Again by taking the 
adjoints, we have $U T^{-1}=T^{*} U$, and hence

$$
T^{*} U^{2}=U^{2} T^{*}
$$

It follows by an argument similar to that of W. A. Beck and C. R. Putnam [1] that

$$
U T^{*}=T^{*} U
$$

Hence from (1) and (2), $T^{*} U=T^{-1} U$ which implies that $T$ is unitary. The authors would like to thank the referee for his suggestions to improve the original text of the paper. The second author wishes to express her thanks to Dr. B. S. Yadav for his valuable help and kind suggestions in the preparation of this paper.

\section{REFERENCES}

1. W. A. Beck and C. R. Putnam, A note on normal operators and their adjoints, J. London Math. Soc. 31 (1956), 213-216. MR 17, 1114.

2. S. K. Berberian, The numerical range of a normal operator, Duke Math. J. 31 (1964), 479-483. MR 29 \#1539.

3. P. R. Halmos, A Hilbert space problem book, Van Nostrand, Princeton, N.J., 1967. MR 34 \#8178.

4. J. P. Williams, Operators similar to their adjoints, Proc. Amer. Math. Soc. 20 (1969), 121-123. MR 38 \#1552.

Faculty of Mathematics, University of Delhi, Delhi 7, India 